\title{
Anomalies in the decay rates of antiprotonic helium-atom states
}

\author{
H. Yamaguchi, T. Ishikawa, J. Sakaguchi, and E. Widmann \\ Department of Physics, University of Tokyo, 7-3-1 Hongo, Bunkyo-ku, Tokyo 113-0033, Japan
}

J. Eades, R. S. Hayano, and M. Hori

CERN, CH-1211 Geneva 23, Switzerland

H. A. Torii

Institute of Physics, University of Tokyo, Komaba, Meguro-ku, Tokyo 153-8902, Japan

B. Juhász

Institute of Nuclear Research of the Hungarian Academy of Sciences, H-4001 Debrecen, Hungary

D. Horváth

KFKI Research Institute For Particle and Nuclear Physics, H-1525 Budapest, Hungary

T. Yamazaki

RI Beam Science Laboratory, RIKEN, Wako, Saitama 351-0198, Japan

(Received 13 February 2002; published 15 August 2002)

\begin{abstract}
Six resonance transitions of the antiprotonic helium atom in helium gas at densities of $3 \times 10^{20}$ $3 \times 10^{21} \mathrm{~cm}^{-3}$ were studied at the antiproton decelerator (AD) of CERN. The decay rates of the daughter states of these transitions were determined either from the time distributions of the resonance spikes or from the widths of the resonance lines. Whereas most of the observed decay rates agree with theoretical calculations of Auger rates, two states, $(n, l)=(37,33)$ and $(32,31)$, were found to have decay rates two orders of magnitude larger than predicted by these calculations. The effect of coupling with near-lying electron-excited states is considered to be the reason for the anomaly of the $(37,33)$ state, as pointed out by Kartavtsev et al. [Phys. Rev. A 61, 062507 (2000)].

DOI: 10.1103/PhysRevA.66.022504

PACS number(s): 36.10.-k, 32.80.Dz
\end{abstract}

\section{INTRODUCTION}

The antiprotonic helium atom $\left(\bar{p}-\mathrm{He}^{+}\right)$is a neutral threebody system consisting of an antiproton, an electron, and a helium nucleus (see [1,2], and a recent comprehensive review, including historical material [3]). Antiprotons captured at high angular momentum have been shown to populate "metastable" states (i.e., states having lifetimes of the order of microseconds) with large principal and angularmomentum quantum numbers $n, l \sim 38$. This metastability results from the drastic dependence of the Auger rate $\gamma_{\mathrm{A}}$ on the lowest possible transition multipolarity $L$, the minimum angular momentum carried away by the Auger electron [4]. Typically

$$
\gamma_{\mathrm{A}} \sim 10^{17-3 L} \mathrm{~s}^{-1} \quad \text { for } \quad L=2,3,4
$$

Antiprotons in metastable states will thus proceed to lower states via radiative transitions along the dominant cascade chain, $(n, l) \rightarrow(n-1, l-1), v \equiv n-l-1=$ const, with typical level lifetimes of $1-1.5 \mu \mathrm{s}[4,5]$. To the extent that the approximation of Eq. (1) is valid, this radiative sequence will continue until the lowest possible multipolarity reaches a critical value $L=3$. At this point Auger decay will dominate and the neutral atom will become a $\bar{p}-\mathrm{He}^{++}$ion, which is then immediately destroyed by Stark transitions.
We arbitrarily refer to states with Auger rates of $10^{8} \mathrm{~s}^{-1}$ or more (i.e., with values much larger than the typical radiative ones) as "short-lived" ones. A laser spectroscopy technique in which a transition from a metastable state to a shortlived state is stimulated as described in Refs. [6,7], has now been applied to many transitions of the $\bar{p}-\mathrm{He}^{+}$atom $[8-11]$.

In 2000, we applied this method to six of the seven transitions shown in Fig. 1 and Table I, using antiprotons produced at the new CERN antiproton facility, the AD (antiproton decelerator). The transition frequencies were determined with accuracies between $0.1 \mathrm{ppm}$ and a few ppm [12]. Except for the 617-nm transition (quantum numbers of the transitions are summarized in Table I), these agreed with threebody variational calculations [13-17] within a few ppm. For the 617-nm transition, the difference from the theoretical value by Korobov [13] was $80 \mathrm{ppm}$. Moreover, its width of $20 \mathrm{GHz}$ [widths quoted in this paper are always full width at half maximum] was clearly inconsistent with the usual figure (1-4 GHz) arising mainly from laser bandwidth. This suggested that its daughter state $(37,33)$ had an anomalously large Auger rate with respect to Eq. (1), the contribution of which to the measured width would normally be negligible (10 MHz) for this $L$.

Another striking deviation from Eq. (1) appeared in the search for the 265-nm transition. We expected that the state $(32,31)$ with $L=4$ would be the lowest metastable one in the $v=0$ chain. However, instead of the 265-nm transition, we 


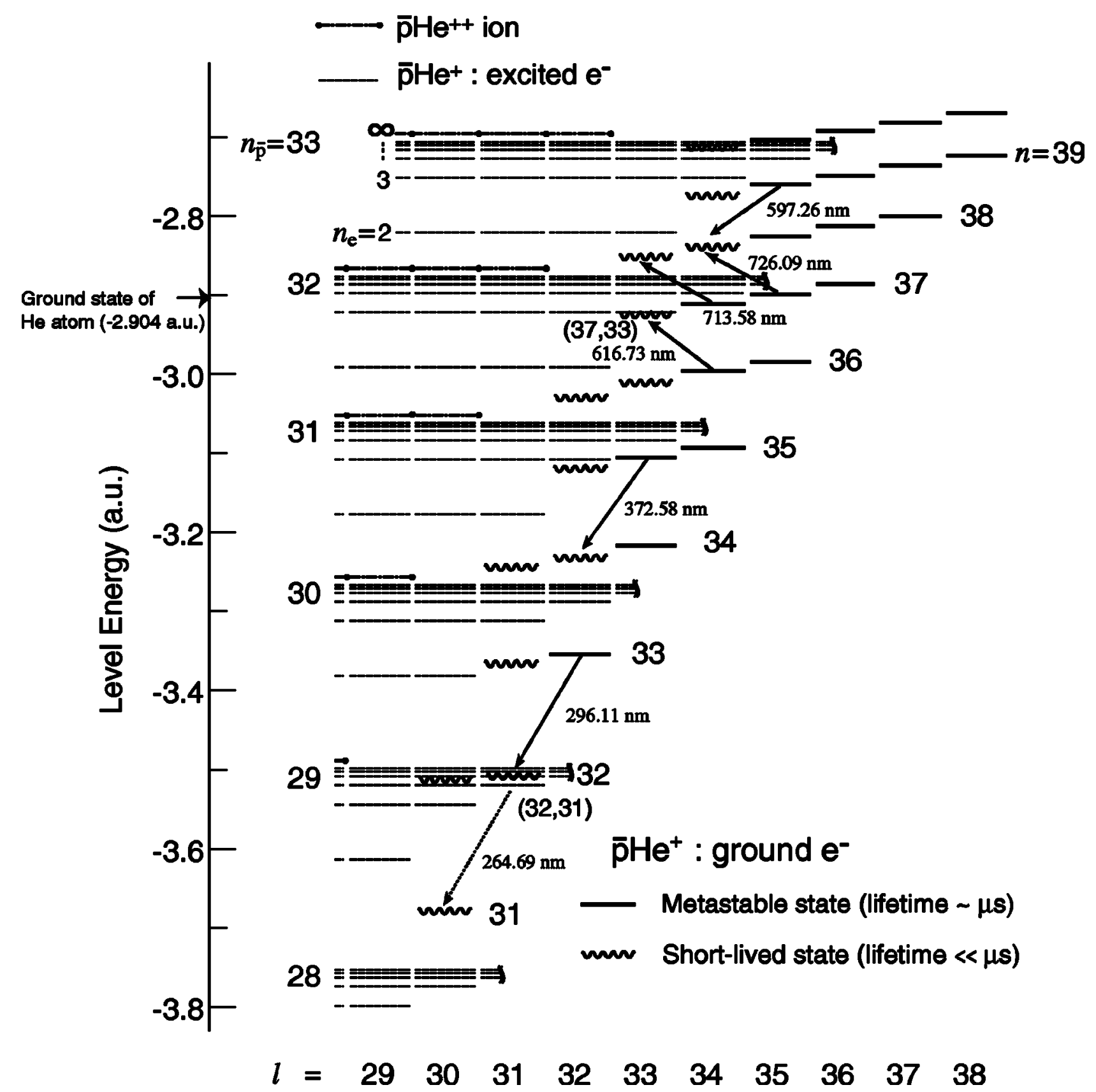

FIG. 1. Energy diagram of $\bar{p}-\mathrm{He}^{+}$atom and $\bar{p}-\mathrm{He}^{++}$ion. The six observed transitions are shown as solid arrows. The 265-nm transition, also shown but as a broken arrow, was not observed in the present work. Two transitions, $597 \mathrm{~nm}$ and $726 \mathrm{~nm}$, have a common daughter state $(38,34)$. The indicated states $(37,33)$ and $(32,31)$ had "anomalous" decay rates. (See text.)

found the 296-nm resonance, near the theoretical wavelength $296.11 \mathrm{~nm}$, proving (a) that the state $(32,31)$ is actually short lived, and (b) that $(33,32)$, and not $(32,31)$, is the lowest metastable state with $v=0$.

It must be mentioned that these conclusions were drawn for certain helium target conditions (density $3 \times 10^{20}$ $3 \times 10^{21} \mathrm{~cm}^{-3}$ ), and that collisional effects are known to change the short-lived or metastable character of certain states [18]. It is thus important to know whether such unexpected short lifetimes were caused by anomalously large Auger rate or by in fact collisional effects.

We discuss in detail below the experimental results on decay rates of daughter states for all six laser resonance tran-
TABLE I. Wavelengths of the transitions. In this paper, transitions are denoted by their wavelengths. The last transition was not observed in the present work.

\begin{tabular}{ccc}
\hline \hline$(n, l) \rightarrow\left(n^{\prime}, l^{\prime}\right)$ & $\Delta v$ & Wavelength $(\mathrm{nm})$ \\
\hline$(37,34) \rightarrow(38,33)$ & 2 & 713.58 \\
$(36,34) \rightarrow(37,33)$ & 2 & 616.73 \\
$(39,35) \rightarrow(38,34)$ & 0 & 597.26 \\
$(37,35) \rightarrow(38,34)$ & 2 & 726.09 \\
$(35,33) \rightarrow(34,32)$ & 0 & 372.58 \\
$(33,32) \rightarrow(32,31)$ & 0 & 296.11 \\
$(32,31) \rightarrow(31,30)$ & 0 & 264.69 \\
\hline \hline
\end{tabular}


sitions mentioned above. The connection of Auger rate anomalies like those described above with electron-excited states (predicted in fact by Kartavtsev et al. [19] before the present experiments were carried out) will be discussed.

\section{EXPERIMENTAL PROCEDURE AND DEDUCTION OF DECAY RATES}

The experimental method, further details of which may be found in Hori et al. [12], was based on the laser spectroscopy technique $[6,7]$ used in similar experiments at the nowdefunct CERN low-energy antiproton ring (LEAR) facility. Antiproton pulses of duration $\sim 300 \mathrm{~ns}$, each of which contained some $10^{7}$ particles were brought to rest in a helium gas target at a temperature of $5.5 \mathrm{~K}$ and pressures between 200 and 2000 mbar (density $3 \times 10^{20}-3 \times 10^{21} \mathrm{~cm}^{-3}$ ). For each transition, measurements were performed at three or four different fixed target pressures within this range. Charged pions produced by their subsequent annihilation were detected by Cerenkov counters, time spectra of these pions being recorded as analog pulses ("ADATS," for analog delayed annihilation time spectrum), by a digital oscilloscope [20].

When we applied a laser pulse tuned to a wavelength of a metastable to short-lived transition, a sharp peak appeared in the ADATS, the intensity (area) of which was proportional to the number of antiproton annihilations induced by the laser pulse $[6,7]$. Spectral line profiles could then be obtained by plotting the peak intensity against the laser wavelength as this was scanned through the central transition wavelength.

Several factors contribute to the observed linewidth. They include the natural Auger width of Eq. (1) as well as Doppler and collisional broadening, and instrumental effects such as the laser bandwidth and power broadening. Most of the line profiles so far observed had widths around $1-2 \mathrm{GHz}$. This is comparable with what would be expected from the Dopplerbroadened instrumental widths alone. However, two of them, the 714-nm and 617-nm resonances had much broader resonance profiles (Fig. 2) suggesting large natural widths of the daughter states due to very fast Auger decays. The first was already known from previous experiments [11]. On the assumption that its measured width is dominated by the Auger width, its fast decay rate $\left(\gamma \sim 5 \times 10^{11} \mathrm{~s}^{-1}\right)$ was determined from the relation between the lifetime $\tau$, the decay rate $\gamma$, and the natural width $\Delta \nu$,

$$
1 / \tau \equiv \gamma=2 \pi \Delta \nu .
$$

This decay rate was in good agreement with the Auger rate expected from Eq. (1) for $L=2\left(\gamma_{\mathrm{A}} \sim 10^{11} \mathrm{~s}^{-1}\right)$. In other words, the decay rate of the $(38,33)$ state is not anomalous.

On the other hand, the width of the newly observed resonance at $617 \mathrm{~nm}$ was $20 \mathrm{GHz}$. This is anomalously broad for $L=3$ transitions, for which the value $\gamma_{\mathrm{A}} \sim 10^{8} \mathrm{~s}^{-1}$ obtained from Eq. (1) corresponds to a width of the order of $10 \mathrm{MHz}$ (as mentioned above).

For these two transitions we fitted the resonance profiles with a sum of two Voigt functions. A Voigt function is a convolution of a Gaussian and a Lorentzian, thus it has four

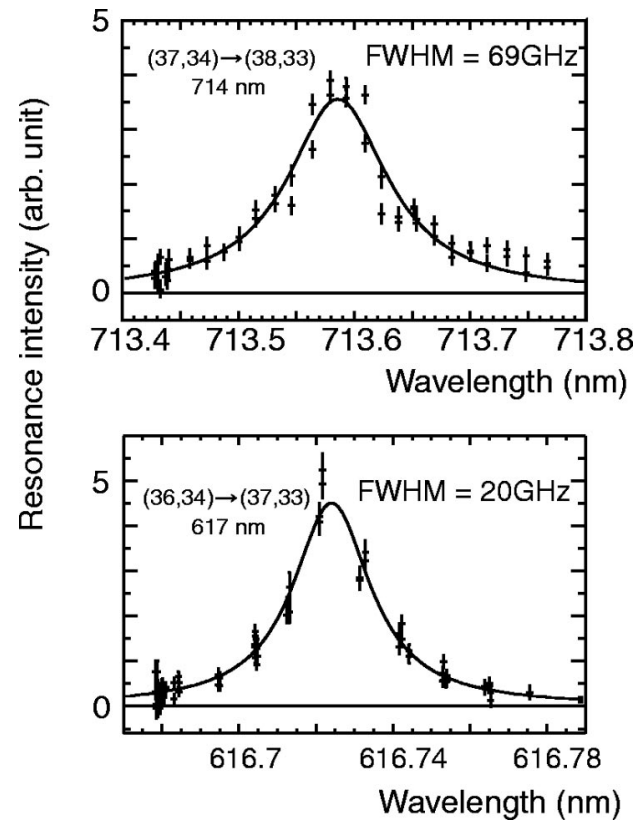

FIG. 2. Resonance profiles of the transitions $(37,34) \rightarrow(38,33)$ and $(36,34) \rightarrow(37,33)$, with target helium gas conditions $5.5 \mathrm{~K}$ and 260 mbar.

parameters, which are, Gaussian width, Lorentzian width, central frequency, and height. The two fitted functions have identical shape but their centers are separated by the hyperfine splitting [21], caused by the coupling of the electron spin and the $\bar{p}$ orbital magnetic moment.

The Gaussian width includes contributions from the laser linewidths and the Doppler broadening. Laser linewidths were determined by measurement [12] to be $3-5 \mathrm{GHz}$ for these cases, while the Doppler widths were calculated assuming Maxwell-Boltzmann velocity distribution of the atoms. The Lorentzian width, then obtained from the fit could be converted to values for the natural widths/decay rates of the daughter states using Eq. (2).

Table II shows various contributions to the total width of the 714-nm and 617-nm resonances. For the hyperfine split-

TABLE II. Various contributions to the width (in $\mathrm{GHz}$ ) of the 714-nm and 617-nm resonances. Power broadening and collisional broadening are less than $1 \mathrm{GHz}$. The typical order of Auger widths, estimated by Eq. (1), are also shown for the purpose of comparison.

\begin{tabular}{lcc}
\hline \multicolumn{1}{c}{ Transition } & $714 \mathrm{~nm}$ & $617 \mathrm{~nm}$ \\
\hline Total experimental width & $69 \pm 2$ & $20 \pm 1$ \\
Broadening source & & \\
$\quad$ in addition to the natural width & & \\
Laser linewidth & $4 \pm 1$ & $4 \pm 1$ \\
Doppler width & 0.3 & 0.4 \\
Power broadening & $\ll 1$ & $\ll 1$ \\
Collisional broadening & $\ll 1$ & $\ll 1$ \\
Hyperfine splitting [22] & 1.7 & 1.7 \\
Deduced natural width & $61 \pm 4$ & $18 \pm 3$ \\
Typical Auger width & 16 & 0.016 \\
\hline \hline
\end{tabular}




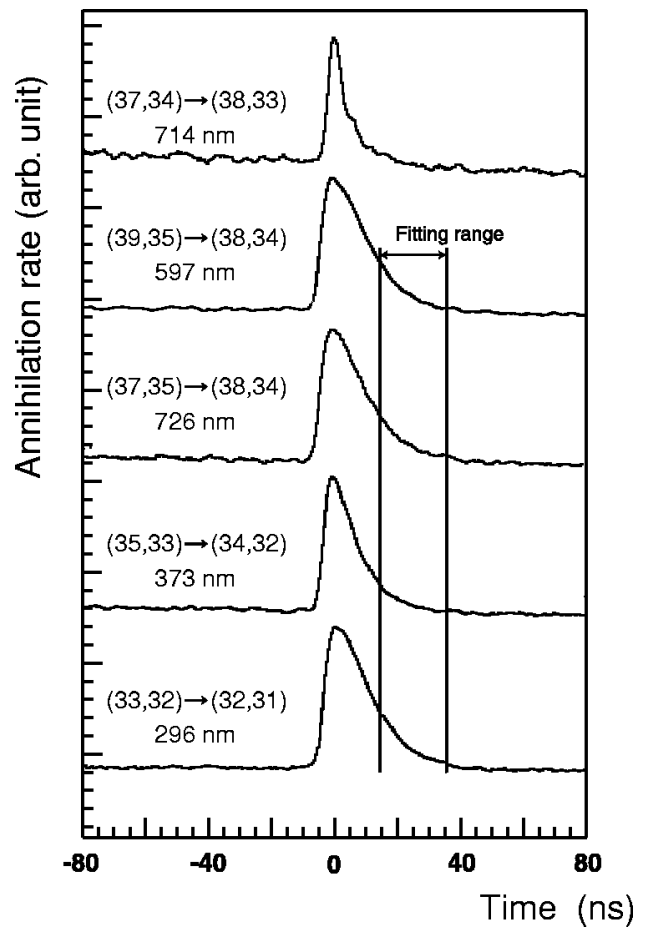

FIG. 3. Comparison of the resonance peaks in ADATS. For each transition, typically spectra of about $100 \mathrm{AD}$ shots are summed up. The peak heights are normalized to the same value.

tings, theoretical values by Bakalov and Korobov [22] were used. In general, the remaining Lorentzian part includes contributions from the collisional and power broadening effects as well as the natural width. However, in both cases the Lorentzian part showed little or no change when we varied the target gas density, which means the collisional and power broadening effects were much less than $1 \mathrm{GHz}$. We consequently assumed that the natural and Lorentzian widths were approximately equal. Most of the errors come from the uncertainty of the laser linewidth and the fit error.

For the remaining four resonances the daughter state Auger widths are much smaller than the instrumental widths, and do not significantly influence the observed line shape. Their corresponding lifetimes are, however, long enough to produce tails in the ADATS resonance peaks themselves. The time profile of the resonance peak intensity $I(t)$ can be represented as a convolution of $I_{\mathrm{i}}(t)$, which is the instrumental time profile of the resonance peak, and an exponential function $\exp (-\gamma t)$. Here $I_{\mathrm{i}}(t)$ is determined by the time profile of the laser pulse ( $3-5 \mathrm{~ns}$ wide) broadened by the transient time spread of the photomultiplier tube (PMT), while the exponential function represents the decay of the daughter state with a rate $\gamma$.

Figure 3 shows the resonance peaks of five transitions. The topmost panel shows the resonance peak of the 714-nm transition discussed above. This is included to exhibit the instrumental shape $I_{\mathrm{i}}(t)$ alone as its 2-ps lifetime is much smaller than the laser-pulse width. In the other four cases, tails corresponding to daughter state lifetimes of order $10 \mathrm{~ns}$ are superimposed on the resonance peaks following $I_{\mathrm{i}}(t)$.

We deduced $\gamma$ in these cases by fitting the convoluted function to the peaks in the small range indicated in the figure. In this range, we assumed $I_{\mathrm{i}}(t)$ is approximately proportional to an exponential function with an instrumental time constant of 3-6 ns, which was determined from the specification of the PMT and Cerenkov counter. The experimental errors were calculated from the statistical errors obtained from the fitting procedure, the uncertainty of the instrumental time constant, and the deviation of the result by a shift of the fit range within \pm 5 ns. For the state $(38,34)$, we used the mean value of the fitting results of two transitions, $597 \mathrm{~nm}$ and $726 \mathrm{~nm}$.

For these four states, measurements were done at three or four target densities between $3 \times 10^{20}$ and $3 \times 10^{21} \mathrm{~cm}^{-3}$, and revealed no significant density dependence of Auger rate. However, it might be interesting to see if there are dependence for far lower densities.

\section{RESULTS AND DISCUSSION}

\section{A. Energy-level diagram}

Figure 1 shows the energy levels of antiprotonic helium atom $\bar{p}-\mathrm{He}^{+}$, with solid lines denoting metastable states and wavy lines denoting short-lived, or Auger-dominated ones. The metastable zone is shown on the right as in previous publications (e.g., [12]), and includes energy levels corresponding to a $1 s$ "ground" electron $\left(n_{e}=1, l_{e}=0\right)$ and an antiproton with $n_{p}^{-}$and $l_{p}^{-}$), of order 35 . We now include on the left some of the electron-excited states $\left(n_{e}=2,3, \ldots\right)$ lying below the $n_{e}=\infty$, i.e., $\bar{p}-\mathrm{He}^{++}$ion states. These electron-excited states are strongly coupled to the continuum, which usually means that precise energy-level calculation is difficult. However, as the electron-excited orbits are far from those of the antiproton for $n_{p}^{-}$smaller than about 40 , their energies can be approximated [19] by

$$
E=-\frac{m_{\bar{p}} m_{\alpha}}{m_{\bar{p}}^{-}+m_{\alpha}} \frac{Z^{2}}{2 n_{\bar{p}}^{2}}-\frac{1}{2 n_{e}^{2}},
$$

in atomic units. Here $Z=2$ is the atomic number of helium. The energies determined from Eq. (3) are shown in the figure (note, however, that the actual energies have a slight dependence on $l_{e}$ and are not perfectly degenerate). The maximum $l_{p}^{-}$and $l_{e}$ being $n_{p}^{-}-1$ and $n_{e}-1$, the maximum $l$ is $n_{p}^{-}+n_{e}$ -2 .

Since these electron-excited states have very short lifetimes, it is not normally necessary to pay any attention to them as they are not directly concerned with the metastability. This ceases to be the case when they intrude into a metastable state, i.e., when, by chance, they are close to it in energy. Among the states observed so far, this degeneracy is close enough to play a role only in the cases of the $(37,33)$ and $(32,31)$ metastable levels.

\section{B. Experimental results}

In Table III and Fig. 4 we summarize the deduced decay rates of the short-lived daughter states and compare them with the Auger values calculated by various authors 
TABLE III. Experimental decay rates and theoretically calculated Auger decay rates in $\mathrm{s}^{-1} . L$ is the minimum transition multipolarity of Auger decay. The results of past experiments [18] are also shown. For the states $(39,35)$ and $(37,34)$, the radiative decay is dominant and the experimental decay rates cannot directly be compared with theoretical Auger rates.

\begin{tabular}{|c|c|c|c|c|c|}
\hline State & $L$ & Experimental values & Korobov and Shimamura [23] & Kartavtsev et al. [19] & Kino [17] \\
\hline$(38,33)$ & 2 & $(3.85 \pm 0.26) \times 10^{11}$ & $3.1 \times 10^{11}$ & $3 \times 10^{11}$ & \\
\hline$(37,33)$ & 3 & $(1.11 \pm 0.16) \times 10^{11}$ & $5.7 \times 10^{9}$ & $3 \times 10^{10}$ & $4.8 \times 10^{10}$ \\
\hline$(38,34)$ & 3 & $(1.11 \pm 0.07) \times 10^{8}$ & $1.3 \times 10^{8}$ & $1.4 \times 10^{8}$ & \\
\hline$(34,32)$ & 3 & $(1.45 \pm 0.16) \times 10^{8}$ & $2.2 \times 10^{8}$ & $2.3 \times 10^{8}$ & \\
\hline$(36,33)$ & 3 & $(2.2 \pm 0.8) \times 10^{8}[18]$ & $2.4 \times 10^{8}$ & & \\
\hline$(32,31)$ & 4 & $(1.17 \pm 0.12) \times 10^{8}$ & $6.1 \times 10^{5}$ & & \\
\hline$(39,35)$ & 4 (radiative) & $(6.5 \pm 0.2) \times 10^{5}[18]$ & $7.0 \times 10^{4}$ & $8 \times 10^{4}$ & \\
\hline$(37,34)$ & 4 (radiative) & $(6.7 \pm 0.5) \times 10^{5}[18]$ & $1.9 \times 10^{5}$ & & \\
\hline
\end{tabular}

$[17,19,23]$. As the radiative rate is comparatively small, the Auger rate is here by far the largest component of the total decay rate. For comparison, we also include alreadypublished data [18] on a few other states, together with their theoretical values.

Most of the measured decay rates are well clustered around the "rule of thumb" relation Eq. (1) between the Auger rate and its minimum multipolarity $L$. They are also close to more precise values from various theoretical studies. States $(37,33)$ and $(32,31)$ are, however, exceptional; their observed rates far exceed both those expected from Eq. (1) and the theoretical values. Let us discuss these two anomalies.

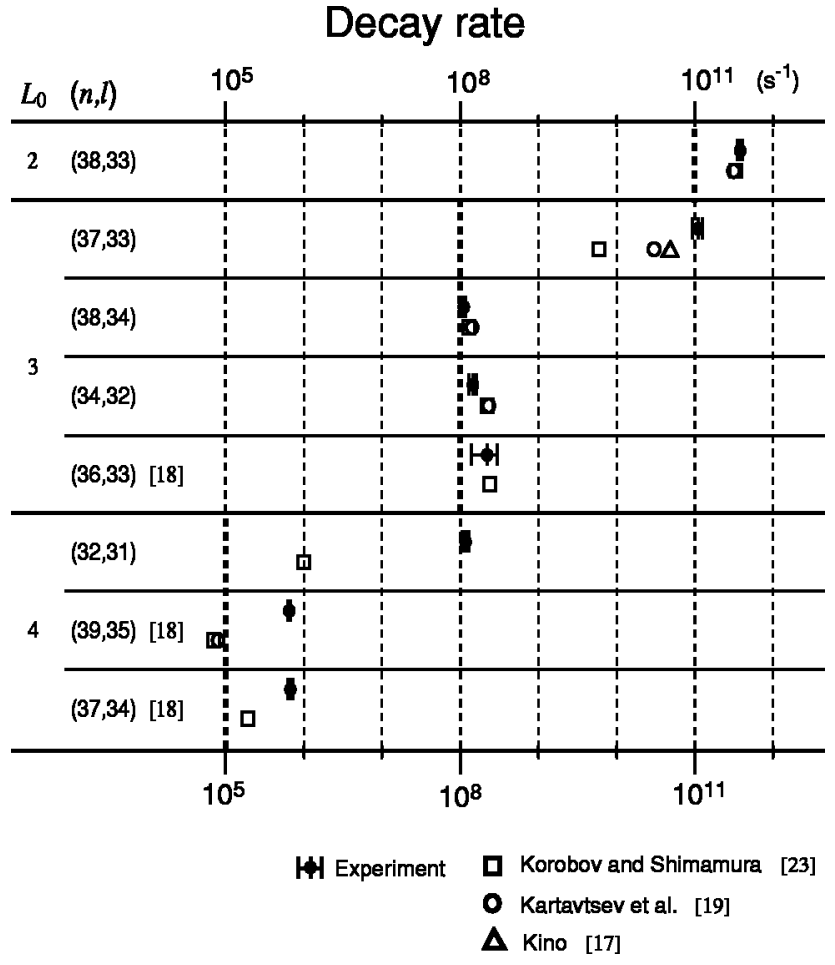

FIG. 4. Decay rate comparison in logarithmic scale. Experimental values and results of three different calculations of Auger rates are shown. $L$ is the minimum transition multipolarity of Auger decay. The rates indicated by bold dotted lines are the orders of magnitude given by Eq. (1) for $L=2,3$, and 4 .
It was pointed out by Kartavtsev and co-workers $[19,24]$ that the $(37,33)$ antiprotonic state with the electron in ground state is strongly coupled with an $l=33$ three-body state of the configuration $(32,31)_{p}^{-} \otimes(3 d)_{e}$, as they have nearly the same energy (cf. Fig. 5). This accidental degeneracy would be expected to give the state $(37,33)$ an anomalous nonradiative character. The admixture of the excited electronic configuration enhances the decay rate, while the dipole transition strength to this state may still be normal for $\Delta v=\Delta(n-l$ $-1)=2$. The value predicted by Kartavtsev and co-workers is in fact fairly close to our observation. Variational calculations by Korobov [13-15], Kino and co-workers [16,17], which include the above configuration mixing effects automatically, yield values that are also close to the experimental ones. Thus, the anomaly in the state $(37,33)$ is very well explained in terms of the mixing effect of the electronexcited configuration.

The second anomaly observed for the state $(32,31)$ is an enhancement of the decay rate of about $10^{2}-10^{3}$ over $0.1-1 \mu^{-1}$ which is expected for an $L=4$ Auger transition. Although a similar configuration mixing effect has been proposed by Kartavtsev and co-workers, the decay rate anomaly has now been shown to be a collisional effect, similar to the lifetime shortening of the state $(37,34)$ [18]. In a future publication [25], we shall present evidence for the collisional origin of the enhanced decay rate at the density used in the presently described measurements, obtained by using a extremely low-density target.

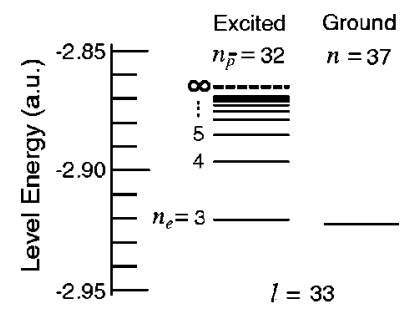

(a)

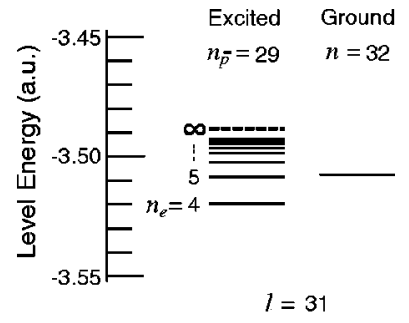

(b)
FIG. 5. Energy diagram of the states $(37,33)$ and $(32,31)$ of normal $\bar{p}-\mathrm{He}^{+}$atom (ground electron configuration), and their neighbor electron-excited states. The energy values were obtained by Eq. (3) and Ref. [13]. 


\section{SUMMARY}

We have studied the short-lived daughter states of six transitions of the antiprotonic helium atom by laser spectroscopy. From the obtained annihilation time spectra and resonance profiles, we deduced their decay rates. We have observed anomalous enhancements of the decay rates in the two states $(37,33)$ and $(32,31)$, which have decay rates more than two orders of magnitude larger than the values expected from their Auger multipolarities. The former case was understood in terms of a mixture of an electron-excited configuration, while the latter is due to collisional effect. The decay rates of three more states, $(38,33),(38,34)$ and $(34,32)$, agree well with the theoretical Auger rates.

\section{ACKNOWLEDGMENTS}

We are grateful to the CERN PS division for their efforts in operating the Antiproton Decelerator and providing us the high-quality antiproton beam, and the CERN cryogenic laboratory for designing the helium target. We acknowledge V. I. Korobov, Y. Kino, O. I. Kartavtsev, and D. D. Bakalov for providing us with the latest theoretical results prior to publications and useful discussions. This work was supported by the Grant-in-Aid for Creative Basic Research (Grant No. 10NP0101) of Monbukagakusho of Japan, and the Hungarian Scientific Research Fund (Grant Nos. OTKA T033079 and TeT-Jap-4/00).
[1] M. Iwasaki, S.N. Nakamura, K. Shigaki, Y. Shimizu, H. Tamura, T. Ishikawa, R.S. Hayano, E. Takada, E. Widmann, H. Outa, M. Aoki, P. Kitching, and T. Yamazaki, Phys. Rev. Lett. 67, 1246 (1991).

[2] T. Yamazaki, E. Widmann, R.S. Hayano, M. Iwasaki, S.N. Nakamura, K. Shigaki, F.J. Hartmann, H. Daniel, T. von Egidy, P. Hofmann, Y.-S. Kim, and J. Eades, Nature (London) 361, 238 (1993).

[3] T. Yamazaki, N. Morita, R.S. Hayano, E. Widmann, and J. Eades, Phys. Rep. 366, 183 (2002).

[4] T. Yamazaki and K. Ohtsuki, Phys. Rev. A 45, 7782 (1992).

[5] I. Shimamura, Phys. Rev. A 46, 3776 (1992).

[6] N. Morita, K. Ohtsuki, and T. Yamazaki, Nucl. Instrum. Methods Phys. Res. A 330, 439 (1993).

[7] N. Morita, M. Kumakura, T. Yamazaki, E. Widmann, H. Masuda, I. Sugai, R.S. Hayano, F.E. Maas, H.A. Torii, F.J. Hartmann, H. Daniel, T. von Egidy, B. Ketzer, W. Müller, W. Schmid, D. Horváth, and J. Eades, Phys. Rev. Lett. 72, 1180 (1994).

[8] R.S. Hayano, F.E. Maas, H.A. Torii, N. Morita, M. Kumakura, T. Yamazaki, H. Masuda, I. Sugai, F.J. Hartmann, H. Daniel, T. von Egidy, B. Ketzer, W. Müller, W. Schmid, D. Horváth, J. Eades, and E. Widmann, Phys. Rev. Lett. 73, 1485 (1994); 73, 3181(E) (1994).

[9] F.E. Maas, R.S. Hayano, T. Ishikawa, H. Tamura, H.A. Torii, N. Morita, T. Yamazaki, I. Sugai, K. Nakayoshi, F.J. Hartmann, H. Daniel, T. von Egidy, B. Ketzer, A. Niestroj, S. Schmid, W. Schmid, D. Horváth, J. Eades, and E. Widmann, Phys. Rev. A 52, 4266 (1995).

[10] H.A. Torii, M. Hori, T. Ishikawa, F.E. Maas, R.S. Hayano, N. Morita, M. Kumakura, I. Sugai, B. Ketzer, H. Daniel, F.J. Hartmann, R. Pohl, R. Schmidt, T. von Egidy, D. Horvath, J. Eades, E. Widmann, and T. Yamazaki, Phys. Rev. A 53, R1931 (1996).

[11] T. Yamazaki, E. Widmann, J. Eades, M. Kumakura, N. Morita, H.A. Torii, M. Hori, T. Ishikawa, F.E. Maas, H. Tamura, R.S.
Hayano, I. Sugai, Y. Fujita, B. Ketzer, H. Daniel, F.J. Hartmann, M. Hasinoff, R. Pohl, R. Schmidt, T. von Egidy, and D. Horváth, Phys. Rev. A 55, R3295 (1997).

[12] M. Hori, J. Eades, E. Widmann, H. Yamaguchi, J. Sakaguchi, T. Ishikawa, R.S. Hayano, H.A. Torii, B. Juhász, D. Horváth, and T. Yamazaki, Phys. Rev. Lett. 87, 093401 (2001).

[13] V.I. Korobov, Phys. Rev. A 54, R1749 (1996).

[14] V.I. Korobov, Nucl. Phys. A 689, 75c (2001).

[15] V.I. Korobov (private communication).

[16] Y. Kino, M. Kamimura, and H. Kudo, Hyperfine Interact. 119, 201 (1999).

[17] Y. Kino (private communication).

[18] M. Hori, H.A. Torii, R.S. Hayano, T. Ishikawa, F.E. Maas, H. Tamura, B. Ketzer, F.J. Hartmann, R. Pohl, C. Maierl, M. Hasinoff, T. von Egidy, M. Kumakura, N. Morita, I. Sugai, D. Horváth, E. Widmann, J. Eades, and T. Yamazaki, Phys. Rev. A 57, 1698 (1998); 58, 1612(E) (1998).

[19] O. Kartavtsev, D. Monakhov, and S. Fedotov, Phys. Rev. A 61, 062507 (2000); 61, 019901(E) (2000).

[20] A. Niestroj, F.J. Hartmann, H. Daniel, B. Ketzer, T. von Egidy, F.E. Maas, R.S. Hayano, T. Ishikawa, H. Tamura, H.A. Torii, N. Morita, T. Yamazaki, I. Sugai, K. Nakayoshi, D. Horváth, J. Eades, and E. Widmann, Nucl. Instrum. Methods Phys. Res. A 373, 411 (1996).

[21] E. Widmann, J. Eades, T. Yamazaki, H.A. Torii, R.S. Hayano, M. Hori, T. Ishikawa, M. Kumakura, N. Morita, I. Sugai, F. Hartmann, T. von Egidy, B. Ketzer, C. Maierl, R. Pohl, and D. Horváth, Phys. Lett. B 404, 15 (1997).

[22] D. Bakalov and V.I. Korobov, Phys. Rev. A 57, 1662 (1998).

[23] V.I. Korobov and I. Shimamura, Phys. Rev. A 56, 4587 (1997).

[24] O.I. Kartavtsev and S.I. Fedotov, in Proceedings of $\mu$ CF01 Conference, Shimoda [Hyperfine Interact. (to be published)].

[25] M. Hori, J. Eades, E. Widmann, H. Yamaguchi, J. Sakaguchi, T. Ishikawa, R.S. Hayano, H.A. Torii, B. Juhász, D. Horváth, and T. Yamazaki (unpublished). 